Review article

\title{
Demystifying endoscopic retrograde cholangiopancreatography (ERCP) during pregnancy
}

\author{
Vítor Magno-Pereira ${ }^{\mathrm{a}, *}$, Pedro Moutinho-Ribeiro ${ }^{\mathrm{b}}$, Guilherme Macedo ${ }^{\mathrm{b}}$ \\ a Gastrenterology Department, Hospital Central do Funchal, Madeira, Portugal \\ ${ }^{\mathrm{b}}$ Gastrenterology Department, Centro Hospitalar de São João, Faculty of Medicine of Porto University, Porto, Portugal
}

\section{A R T I C L E I N F O}

Article history:

Received 5 August 2017

Received in revised form 30 September 2017

Accepted 4 October 2017

Available online $\mathrm{xxx}$

\section{Keywords:}

ERCP

No-radiation

Pregnancy

EUS

Choledocholithiasis

\begin{abstract}
A B S T R A C T
Background: For many years, ERCP was avoided in pregnancy given the concerns regarding the adverse effects that, with special focus on radiation, could occur in the developing fetus. However, the postponement or rejection of ERCP in pregnant women, may lead to a higher risk for mother and fetus, especially when the indication is unequivocal, namely cholangitis, biliary pancreatitis and symptomatic choledocholithiasis.

Summary and key messages: This review aims to summarize the scarce literature on the subject in order to plan ERCP in pregnancy with the highest safety. The use of techniques that reduce radiation and increase the protection of pregnant women allow radiation levels far below the safety limits.

We also discuss the various alternatives of ERCP without radiation. EUS can eliminate the need for ERCP with doubtful choledocholithiasis and plan the best approach in those with previous evidence. The possibility of performing "ERCP" with a linear echoendoscope uniquely under ultrasound control has been described. Conversely, the two-step strategy (initial sphincterotomy with stent placement without fluoroscopy and after delivery, ERCP with lithiasis extraction) proved to be safe obviating fluoroscopy. In conclusion, ERCP can be performed in pregnancy safely and effectively with minimal radiation or even no-radiation at all.
\end{abstract}

(C) 2017 Elsevier B.V. All rights reserved.

\section{Contents}

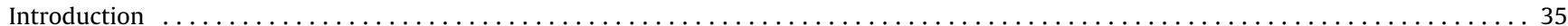

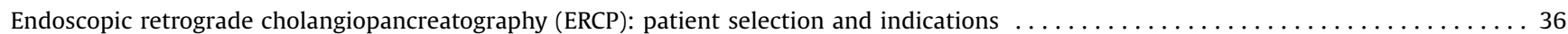

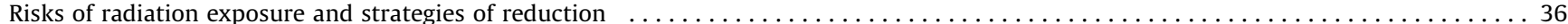

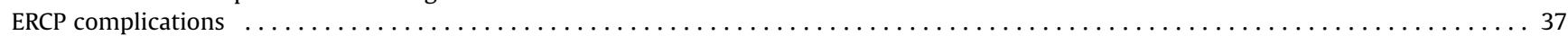

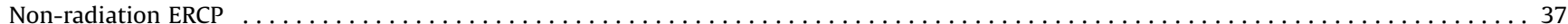

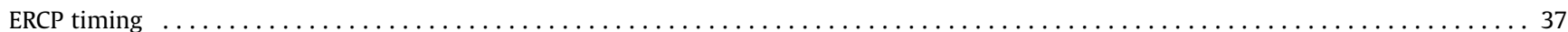

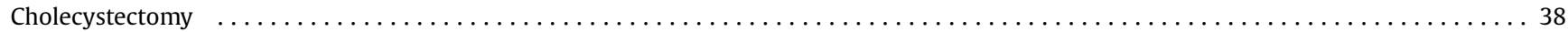

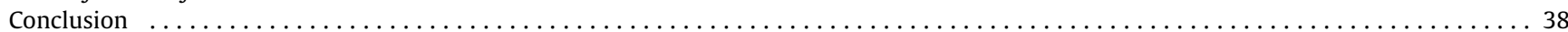

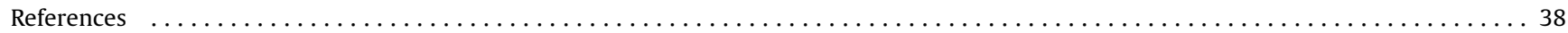

\footnotetext{
* Corresponding author at: Department of Gastroenterology, Hospital Central do Funchal Avenida Luís de Camões, no 57 9004-514 Funchal, Portugal.

E-mail addresses: magnovitorp@gmail.com (V. Magno-Pereira), pmoutinhoribeiro@gmail.com (P. Moutinho-Ribeiro), guilhermemacedo59@gmail.com (G. Macedo).
}

\section{Introduction}

Women are about twice as likely to develop choledocholithiasis compared to men, regardless of the prevalence of cholelithiasis [1]. This discrepancy is more pronounced at younger ages, with a significant reduction in the woman-to-man ratio as the age progresses [2], reflecting the magnitude of the effect of pregnancy and sex hormones.

The litogenicity of female sex hormones is reinforced by studies in which estrogens have been administered to men. In a study with 
patients with prostatic adenocarcinoma, there was an increase in hepatic cholesterol secretion that resulted in an increase in both bile cholesterol saturation and rate of gallstone formation during estrogen treatment [3]. Also, in men with acute myocardial infarction, estrogens increased the risk of biliary lithiasis more than twice [4].

Pregnancy is a major risk factor for biliary lithiasis. The risk increases with frequency and number of pregnancies and reduces with breastfeeding [5]. The risk increases up to 10 times in multiparous compared to nulliparous [6]. During pregnancy there is a decrease in gallbladder motility and a breakdown of cholesterol in bile. These changes are induced by estrogen which increases cholesterol secretion and progesterone which reduces the secretion of bile acids and delays the emptying of the gallbladder. There is also a relative overproduction of hydrophobic bile acids (chenodeoxycholate) which reduces bile's ability to solubilize cholesterol $[1,7]$.

In a prospective ultrasound study with more than 3200 pregnant women without lithiasis (baseline ultrasound), lithiasis or new bile sludge was observed in $7.1 \%$ up to the second trimester, $7.9 \%$ up to the third trimester and $10.2 \%$ up to 6 weeks postpartum. Of the pregnant women with lithiasis or biliary sludge, only $1.2 \%$ developed symptoms of biliary pathology [8]. Up to $10 \%$ of symptomatic pregnant women develop serious complications such as acute cholecystitis, choledocholithiasis or pancreatitis [9].

Risks of ERCP in pregnancy include risks of sedation, radiation or electrocautery to the fetus, as well as technical difficulties related to the changing maternal anatomy and an increased risk to post-ERCP pancreatitis. Therefore we reviewed each potential risk based on the best available evidence to date.

\section{Endoscopic retrograde cholangiopancreatography (ERCP): patient selection and indications}

ERCP is currently established as an essentially therapeutic technique and, in pregnancy, it becomes even more pressing that it is performed for this purpose alone. In this population, it is indicated, as treatment in biliary pancreatitis, symptomatic choledocholithiasis and cholangitis or in the lesions of the pancreatic or biliary duct [10]. The usual risks associated with ERCP, such as perforation, infection, hemorrhage and pancreatitis can have important consequences to both mother and fetus. The fear of inducing irreversible lesions postponed ERCP use for many years in pregnant women.

Nonetheless, it is also relevant to refer that the conservative approach for some of these indications can also be deleterious. As showed in a retrospective study, the conservative management (versus ERCP and/or surgery) of cholelithiasis and its complications in pregnancy is significantly associated with higher recurrent biliary symptoms, number of emergency department visits, number of hospitalizations and cesarean section operations for childbirth [11].

\section{Risks of radiation exposure and strategies of reduction}

Fluoroscopy radiation may have both stochastic effects and deterministic effects. The formers, do not present a dose threshold, the likelihood of developing deleterious effects is proportional to the dose but its severity is dose-independent (e.g. leukemia). In the latters, the dose and severity threshold are proportional to the dose (e.g. cataracts) [12]. Hence the concept "as low as reasonably achievable" radiation has emerged [13]. In fact, the European Society of Digestive Endoscopy (ESGE) recommends in its radiation protection guideline that KAP (kerma-area product) should be monitored, and its cumulative value should be recorded for every ERCP and patient [14].
The American College of Obstetricians and Gynecologists (ACOG) has stated in 2016 diagnostic imaging guidelines that: "Fetal risk of anomalies, growth restriction, or abortion have not been reported with radiation exposure of less than $50 \mathrm{mGy}$, a level above the range of exposure for the diagnostic procedures." ${ }^{15}$ In fact, in a study involving 17 ERCPs in pregnant women with a mean fluoroscopy time of $14 \mathrm{~s}$ (range 1-48s), the estimated fetal radiation exposure was $0.40 \mathrm{mGy}$ (range $0.01-1.8 \mathrm{mGy}$ ). There was a correlation between fluoroscopy time and radiation exposure, but there was a wide range of exposure for individual fluoroscopy times [16]. In another study, the estimated fetal radiation was $1.02-5.77 \mathrm{mGy}(0.00102-0.00577 \mathrm{~Gy})$ [17]. The International Commission on Radiological Protection (ICRP) recommends monitoring fetal radiation when a dose is expected to exceed $0.01 \mathrm{~Gy}$ [18] Studies in clinical practice have estimated fetal radiation induced by ERCP of $<0.1-5.77 \mathrm{mGy}(0.0001-$ $0.006 \mathrm{~Gy}$ ). Although measurements show low fetal absorbed dose values (clearly below ICRP radiation cutoff), methodologies also demonstrate that minute variations in the procedure including degree of incidence of fluoroscopy, position of the patient, orientation of the fetus and endoscopist experience can have dramatic effects on the final dose absorbed [19].

The risk to the fetus is also dependent on the gestational age. Based on data from atomic bomb survivors, it appears that the risk to the central nervous system is greatest when the exposure occurs at 8-15 weeks of gestation. It has been suggested that the minimum dose for this adverse effect might be $60-310 \mathrm{mGy}$ but the lowest recorded dose to induce severe intellectual disability was $610 \mathrm{mGy}$. After 16 weeks there is a low risk for intellectual disability. [15]

In a study involving 23 pregnant patients submitted to a total of 29 ERCPS, 3 women did not know that they were pregnant [20]. It is important to confirm with all women of childbearing age if they are pregnant at the time of the procedure due to the risks in early pregnancy. Before implantation ( $0-2$ weeks after conception), with a minimum dose of 50-100 mGy the effect can be "all or none": death of embryo or no consequence. During organogenesis (2-8 weeks) the estimated threshold dose is $200 \mathrm{mGy}$ for congenital anomalies (skeleton, eyes, genitals) and growth restriction [15].

Fetal radiation exposure depends on multiple factors such as size and body composition of the mother, gestational age as discussed, position of the mother and fetus and exposure techniques. The use of a lead apron placed inferiorly to the pelvis and lower abdomen of the pregnant woman is recommended although most of the exposure of fetal radiation comes from radiation diffused by the mother, so it is essential to complement this protection with other strategies [16].

There are several strategies to reduce the radiation exposure to the mother and fetus during fluoroscopy: use as little fluoroscopy time and obtain as few spot exposures as possible; keep the image intensifier as close to the patient as possible; use Boost Mode and Magnification Mode only when necessary; use a modern fluoroscopy equipment; collimate $\mathrm{x}$-ray beam to the area of interest and use a low frame-rate. In manual mode, use higher $\mathrm{kV}$ (at least 75) and lower $\mathrm{mA}$ settings (decrease in patient dose of $50 \%$ can be achieved by increasing voltage from $75 \mathrm{kV}$ to $96 \mathrm{kV}$ ). Monitoring and recording the amount of fluoroscopy time is another strategy to increase awareness of the endoscopist and reduce the total exposure time [13].

Amniotic fluid is a possible conductor of current to the fetus. Thus, the uterus should not be between the grounding pad and the electrical catheter. The pad should be placed higher in the posterior thoracic wall (rather than the hip). Bipolar electrocautery should be preferred, to minimize this risk [10].

National Radiological Protection Board advises magnetic resonance imaging (MRI) avoidance during the first trimester 
due to limited experience. It stated that it may be used only after critical risk-benefit analysis and only if the diagnostic information cannot be provided by ultrasound [21]. This recommendation is based on theoretical possible biological effects and there are no harmful reports of MRI during pregnancy known until the date of writing.

\section{ERCP complications}

A retrospective cohort study of the National Inpatient Sample with 907 pregnant and 2721 non-pregnant women demonstrated that there was no difference in rates of perforation, infection and bleeding of ERCPs performed in pregnant women. Post-ERCP pancreatitis (PEP) occurred in $12 \%$ of pregnant women vs. $5 \%$ nonpregnant. Pregnancy was an independent risk factor (OR 2.8, CI 2.1-3.8) for PEP [22]. The pregnancy group had less pancreatic stents placed than the control group, which was statistically significant. Nevertheless, even after adjusting for the stents, pregnancy was still an independent factor. The authors suggested several possible mechanisms for this: the tendency to use the least radiation possible could lead to more difficult cannulation; the physicians could be less prone to give large volumes of intravenous fluid during/after ERCP or to treat with NSAIDs (nonsteroidal antiinflammatory drugs) which can be teratogenic. There could also be a physiologic mechanism that inherently predisposed pregnant women to PEP. However, the authors emphasize that ERCP should not be discouraged in pregnant women with clear indication. It is still the least-invasive approach to therapy for bile duct pathology (vs. surgical or radiologic management).

Another unicenter retrospective study had previously suggested this hypothesis with PEP in 16\% of the 68 ERCPs performed on 65 pregnant [23].

\section{Non-radiation ERCP}

In a retrospective study, 21 ERCPs without fluoroscopy were analyzed in pregnant women [24]. A previous imaging study was performed (all had abdominal ultrasound, 6 echoendoscopy (EUS) and 4 magnetic resonance cholangiopancreatography (MRCP)). The bile cannulation was confirmed by the observation of bile around the guidewire (with forward and backward movements). When bile was not visible, a 5 French ( $\mathrm{Fr}$ ) $2 \mathrm{~cm}$ stent was placed. In the case of drainage of bile by the stent, sphincterotomy was performed with needle-knife followed by cannulation and stent removal. In case of drainage of pancreatic juice, access papillotomy was performed. Peroral cholangioscopy (Spyglass ${ }^{\mathbb{R}}$ ) was used to confirm technical success in 5 cases without previous EUS/MRCP. One case of PEP was reported (catheter advancement prior to cannulation confirmation) but there was no symptomatic recurrence in any of the cases.

A two stage ERCP approach has been described in a study involving 11 pregnant women [25]. ERCP was performed without fluoroscopy or EUS. All the women presented with jaundice and pain, registering 2 cases of cholangitis. In a first time, the initial sphincterotomy was performed with stent placement without using fluoroscopy. After delivery, ERCP was performed with lithiasis extraction. In this second ERCP, 1 case presented no lithiasis, 8 cases with stones with $5-8 \mathrm{~mm}$ removed with Dormia basket, and 2 cases with stones $>15 \mathrm{~mm}$ ( 1 resolved with extracorporeal lithotripsy and 1 surgically). All deliveries were of term, with no need for rehospitalization and no changes in infant development during the follow-up period up to 6 years. A similar two-stage intervention was carried in a retrospective study involving 17 third-trimester pregnancies (34 ERCPS): 7 cases with endoscopic nasobiliary drainage and 10 with bile duct plastic stent [26]. There were two complications, namely, one biliary tract hemorrhage and one case of acute mild pancreatitis. All symptoms were significantly alleviated. Eleven cases had term labors and 6 had premature delivery.

The strategy of only placing a stent has the advantage of fluoroscopy being minimal but carries a risk of migration or occlusion of the stent that could cause cholangitis and need for another ERCP. Performing only sphincterotomy facilitates the passage of stones and is preferable to stent only but might not be sufficient to maintain decompression until postpartum cholecystectomy [25].

In a retrospective study, Vohra et al. demonstrated the successful single-session endosonography-based ERCP without fluoroscopy in 10 pregnant patients with suspected choledocolithiasis [27]. IDUS (miniprobe intraductal ultrassound) was not used given the need for fluoroscopy. EUS excluded choledocholithiasis in 4 cases, thus avoiding ERCP. Lithiasis was confirmed in the remaining 6 cases, the number of bile stones extracted being equal to the number visualized in the EUS. Bile cannulation was confirmed by aspiration of bile. A numbered guidewire was used to monitor the progression inside the bile duct endoscopically. Biliary sphincterotomy with endocut mode was performed, followed by stone extraction using a standard Dormia basket. Cholangioscopy was used in two patients given the fragmentation of lithiasis to confirm complete extraction. There were no complications, repetition of procedures, adverse events or abortions.

These results support the studies of single session EUS-ERCP in non-pregnant patients, demonstrating that ERCP can be safely avoided in a significant number of patients (44-75\%, depending on the risk stratification for suspected choledocholithiasis of the patients) [28,29].

Nonradiation ERCP with endoscopic biliary sphincterotomy plus papillary balloon dilation was retrospectively evaluated for the treatment of choledocholithiasis in 22 pregnant patients. Balloon dilation was performed with a $6-\mathrm{mm}$ balloon in 17 patients and an 8-mm in five patients, according to the diameter of the CBD (common bile duct) on US (ultrasonography) or MRCP, with effective stone extraction in 18 patients. No biliary stent was used. All patients delivered at term, and none experienced recurrence of symptoms during the 6 -month follow-up. There were 2 cases of PEP [30].

Failed cannulation while performing ERCP during pregnancy is a technically demanding situation. There has been a case report of a successfully attempted EUS guided rendezvous technique, with fluoroscopy used only to confirm the exit of the wire from ampulla, wire reposition in intrahepatic biliary system and the position of the stent. The whole fluoroscopy exposure time was $3 \mathrm{~s}$ [31].

\section{ERCP timing}

There is the generic recommendation to avoid endoscopy in the first trimester whenever possible [10]. In ERCP, there has also been worries during the first trimester. In a retrospective review of 68 ERCPs performed in 65 pregnant patients, there were no perforation, sedation adverse events, postsphincterotomy bleeding, cholangitis, or procedure-related maternal or fetal deaths. However, women submitted to ERCP in the first trimester had the lowest percentage of term pregnancies (73\%), the highest risk of preterm delivery (20\%) and low birth weight newborns (21\%) [23]. However, the authors suggested that it was the development of hepatobiliary disease in the first trimester of pregnancy that could be associated with preterm delivery or low birth weight and not the procedure itself. They suggested therefore "to intervene appropriately as early as possible".

In fact, as showed in a retrospective study of 112 pregnant women, the conservative management (versus ERCP and/or surgery) of cholelithiasis and its complications (mainly biliary 
colic, biliary pancreatitis, acute cholecystitis and choledocholithiasis) in pregnancy is significantly associated with higher cesarean section operations for childbirth and higher recurrent biliary symptoms, number of emergency department visits and number of hospitalizations [11]. ERCP was performed at a mean of 3.7 days after the onset of the symptoms by experienced endoscopists. CBD cannulation rate was $100 \%$ and biliary sphincterotomy was done in $88 \%$. Only one complication was reported, one case of PEP that resolved in a few days with conservative management. But it is important to refer that this study included only 4 ERCP in the first trimester. Another study, demonstrated that up to $72 \%$ of pregnant women with biliary pancreatitis will have an intercurrence during pregnancy if a conservative approach is taken [32].

In a retrospective matched-cohort study with 907 ERCP performed in pregnant women, there is no reference to the trimesters or week of gestation. Nevertheless, there were no differences in maternal mortality, fetal distress or fetal loss when compared to age-matched pregnant women. Curiously, the risk of preterm labor in pregnant patients undergoing ERCP was lower than the national average [22].

Finally, the evidence is scarce relating specifically to the best timing for each indication. Taking into account the aforementioned studies, ERCP should not be deferred in cases of cholangitis, biliary pancreatitis and symptomatic choledocholithiasis. Each case should be discussed with the obstetrician to proceed with ERCP even in the first trimester. Lesions of the pancreatic or biliary duct should be evaluated case-by-case in terms of urgency of the procedure. When the event occurs in the third trimester, the timing of delivery should be discussed with the obstetrician to decide between an early delivery and ERCP post-partum or ERCP in the third trimester, considering that ERCP is a safe procedure in third trimester.

\section{Cholecystectomy}

Surgery during pregnancy increases the risk of fetal loss. Therefore, the indication is usually limited to urgent situations as acute cholecystitis. The second trimester is considered to be the optimal time for cholecystectomy, with the lowest risk for fetal morbity [33]. However, there are also several studies demonstrating the safety in urgent cases during the first trimester [34]. Surgery in the third trimester was generally limited for fear of induction of preterm labor (up to $40 \%$ in initial studies). Most recent studies limited to laparoscopic approach have shown that surgery is safe in any trimester without significant increased risk [35].

Women who have complicated gallstone are unlikely submitted to cholecystectomy during pregnancy. Nevertheless, the risk of symptom recurrence either during pregnancy or in the early postpartum is higher when cholecystectomy is not performed. ERCP with biliary sphincterotomy significantly reduces this risk [36]. Cholecystectomy should be considered in complicated gallstone disease during pregnancy, especially in patients that do not undergo or fail ERCP with biliary sphincterotomy. The value of intrapartum cholecystectomy after ERCP has not been specifically studied, but the small studies of ERCP during pregnancy previously stated suggest that endoscopic sphincterotomy may be sufficient to prevent recurrence during pregnancy $[24,25,30]$. Likewise, laparoscopic cholecystectomy could be safely done immediately postpartum in these patients.

\section{Conclusion}

ERCP can be performed in pregnancy safely and effectively in women who have a clear indication. There are multiple strategies for reducing maternal and fetal risks associated with this technique.

EUS can eliminate the need for ERCP and its risks in pregnant women with no concrete evidence of choledocholithiasis, and even plan the best approach in those that present previous evidence by specifying the number, size and location of the lithiasis. EUS-CPRE thus presents itself as the ideal approach in pregnancy. The use of a linear EUS scope for the entire procedure has already been described. Also, the two-step strategy proved to be a safe and effective alternative without the need for fluoroscopy. However, stent placement increases the risk of recurrence of ERCP, so the risks of repetition of the procedure should be weighed against controlled radiation exposure from a single ERCP. Whichever method is used, it should be combined with other radiation reduction strategies in ERCP, namely: a lead apron placed inferiorly to the pelvis and lower abdomen of the pregnant; as little fluoroscopy time and as few spot exposures as possible; image intensifier as close to the patient; dismiss boost and magnification modes; collimate x-ray beam and use low frame-rate; use higher $\mathrm{kV}$ and lower $\mathrm{mA}$ settings. Monitoring and recording the amount of fluoroscopy time is encouraged and the procedure should be done by an experienced endoscopist.

There are no prospective studies to quantify the superiority of EUS-ERCP in the different outcomes and demonstrate costeffectiveness versus other approaches. However, given the small series and the rarity of the procedure compared to other populations, this is a level of evidence difficult to achieve.

\section{References}

[1] Everson GT. Pregnancy and gallstones. Hepatology 1993;17(1):159-61.

[2] The epidemiology of gallstone disease in rome, Italy. Part I. prevalence data in men. The Rome group for epidemiology and prevention of cholelithiasis (GREPCO). Hepatology 1988;8(4):904-6.

[3] Henriksson P, Einarsson K, Eriksson A, Kelter U, Angelin B. Estrogen-induced gallstone formation in males: relation to changes in serum and biliary lipids during hormonal treatment of prostatic carcinoma. J Clin Invest 1989;84 (3):811-6.

[4] Gallbladder disease as a side effect of drugs influencing lipid metabolism. Experience in the Coronary Drug Project. N Engl J Med 1977;296(21):1185-90.

[5] Liu B, Beral V, Balkwill A. Childbearing, breastfeeding, other reproductive factors and the subsequent risk of hospitalization for gallbladder disease. Int J Epidemiol 2009;38(1):312-8.

[6] Adilson CunhaFerreira FMF, Marum Mauad Fernando, Gadelha Antônio, Spara Patrícia, Filho Isac Jorge. Clinical and ultrasonographic risk factors associated with asymptomatic gallstones in women. Radiol Bras 2004;37(2):77-82.

[7] Kern Jr. F, Everson GT, DeMark B, et al. Biliary lipids, bile acids, and gallbladder function in the human female: effects of pregnancy and the ovulatory cycle. J Clin Investig 1981;68(5):1229-42.

[8] Ko CW, Beresford SA, Schulte SJ, Matsumoto AM, Lee SP. Incidence, natural history, and risk factors for biliary sludge and stones during pregnancy. Hepatology 2005;41(2):359-65.

[9] Valdivieso V, Covarrubias C, Siegel F, Cruz F. Pregnancy and cholelithiasis: pathogenesis and natural course of gallstones diagnosed in early puerperium. Hepatology 1993;17(1):1-4

[10] Committee A.SoP. Shergill AK, Ben-Menachem T, et al. Guidelines for endoscopy in pregnant and lactating women. Gastrointest Endosc 2012;76 (1):18-24.

[11] Othman MO, Stone E, Hashimi M, Parasher G. Conservative management of cholelithiasis and its complications in pregnancy is associated with recurrent symptoms and more emergency department visits. Gastrointest Endosc 2012;76(3):564-9.

[12] Raijman I. Performing endoscopic retrograde cholangiography without radiation exposure: are we ready for it? Gastrointest Endosc 2016;84 (5):770-2.

[13] Amis ES. Risks of radiation exposure in the endoscopy suite: principles, cautions, and risks to patients and endoscopy staff. Techn Gastrointest Endoscopy 2007:9(4):213-7.

[14] Dumonceau JM, Garcia-Fernandez F, Verdun F, et al. Radiation protection in digestive endoscopy: European society of digestive endoscopy (ESGE) guideline. Endoscopy 2012;44(04):408-24.

[15] Committee Opinion No. 656: guidelines for diagnostic imaging during pregnancy and lactation. Obstet Gynecol 2016;127(2):e75-80.

[16] Kahaleh M, Hartwell GD, Arseneau KO, et al. Safety and efficacy of ERCP in pregnancy. Gastrointest Endoscopy 2004;60(2):287-92.

[17] Tham T. Safety of ERCP during pregnancy. Am J Gastroenterol 2003;98(2):30811. 
[18] Protection ICoR. Pregnancy and medical radiation. Ann ICRP 200084(30).

[19] Huda A, Garzon WJ, Filho GC, et al. Evaluation of staff, patient and fetal radiation doses due to endoscopic retrograde cholangiopancreatography (ERCP) procedures in a pregnant patient. Radiat Prot Dosimetry 2016;168 (3):401-7.

[20] Jamidar PA, Beck GJ, Hoffman BJ, et al. Endoscopic retrograde cholangiopancreatography in pregnancy. Am J Gastroenterol 1995;90(8):1263-7.

[21] Board NRP. Protection of pregnant patients during diagnostic medical exposures to ionising radiation. Doc NRPB 2009;3-16.

[22] Inamdar S, Berzin TM, Sejpal DV, et al. Pregnancy is a risk factor for pancreatitis after endoscopic retrograde cholangiopancreatography in a national cohort study. Clin Gastroenterol Hepatol 2016;14(1):107-14.

[23] Tang SJ, Mayo MJ, Rodriguez-Frias E, et al. Safety and utility of ERCP during pregnancy. Gastrointest Endosc 2009;69(Pt. 1 (3)):453-61.

[24] Shelton J, Linder JD, Rivera-Alsina ME, Tarnasky PR. Commitment, confirmation, and clearance: new techniques for nonradiation ERCP during pregnancy (with videos). Gastrointest Endosc 2008;67(2):364-8.

[25] Sharma SS, Maharshi S. Two stage endoscopic approach for management of choledocholithiasis during pregnancy. J Gastrointest Liver Dis 2008;17 (2):183-5.

[26] Zhou Y, Zhang X, Zhang X, et al. ERCP in acute cholangitis during third trimester of pregnancy. Hepatogastroenterology 2013;60(125):981-4.

[27] Vohra S, Holt EW, Bhat YM, Kane S, Shah JN, Binmoeller KF. Successful singlesession endosonography-based endoscopic retrograde cholangiopancreatography without fluoroscopy in pregnant patients with suspected choledocholithiasis: a case series. J Hepatobil Pancreat Sci 2014;21(2):93-7.
[28] Lee YT, Chan FK, Leung WK, et al. Comparison of EUS and ERCP in the investigation with suspected biliary obstruction caused by choledocholithiasis: a randomized study. Gastrointest Endosc 2008;67(4):660-8.

[29] Sharma R, Menachery J, Choudhary NS, Kumar M, Puri R, Sud R. Routine endoscopic ultrasound in moderate and indeterminate risk patients of suspected choledocholithiasis to avoid unwarranted ERCP: a prospective randomized blinded study. Indian J Gastroenterol 2015;34(4):300-4.

[30] Ersoz G, Turan I, Tekin F, Ozutemiz O, Tekesin O. Nonradiation ERCP with endoscopic biliary sphincterotomy plus papillary balloon dilation for the treatment of choledocholithiasis during pregnancy. Surg Endosc 2016;30 (1):222-8.

[31] Singla V, Arora A, Tyagi P, Sharma P, Bansal N, Kumar A. Failed common bile duct cannulation during pregnancy: rescue with endoscopic ultrasound guided rendezvous procedure. Endosc Ultrasound 2016;5(3):201-5.

[32] Swisher SG, Hunt KK, Schmit PJ, Hiyama DT, Bennion RS, Thompson JE. Management of pancreatitis complicating pregnancy. Am Surg 1994;60 (10):759-62.

[33] Neudecker J, Sauerland S, Neugebauer E, et al. The European Association for Endoscopic Surgery clinical practice guideline on the pneumoperitoneum for laparoscopic surgery. Surg Endosc 2002;16(7):1121-43.

[34] Cosenza CA, Saffari B, Jabbour N, et al. Surgical management of biliary gallstone disease during pregnancy. Am J Surg 1999;178(6):545-8.

[35] Jackson H, Granger S, Price R, et al. Diagnosis and laparoscopic treatment of surgical diseases during pregnancy: an evidence-based review. Surg Endosc 2008;22(9):1917-27.

[36] Veerappan A, Gawron AJ, Soper NJ, Keswani RN. Delaying cholecystectomy for complicated gallstone disease in pregnancy is associated with recurrent postpartum symptoms. J Gastrointest Surg 2013;17(11):1953-9. 